\title{
Personalized Activity Based eLearning
}

\author{
Ian O'Keeffe, Athanasios Staikopoulos, Rachael Rafter, Eddie Walsh, Bilal Yousuf, \\ Owen Conlan and Vincent Wade \\ Knowledge and Date Engineering Group \\ School of Computer Science and Statistics, Trinity College Dublin, Ireland \\ \{lan.OKeeffe,Athanasios.Staikopoulos, Rachael.Rafter,Eddie.Walsh,yousufbi, \\ Owen.Conlan,Vincent.Wade\}@scss.tcd.ie
}

\begin{abstract}
The aim of personalizing web information systems is to tailor content (media) to the user's personal preferences, goals and context, in turn increasing the reusability of that content. However, most developers are increasingly seeking to apply 'Web as a Platform' based approaches where webbased content is integrated with web services to provide the platform and environment features that were traditionally offered by desktop environments and applications. Therefore, next generation personalized web information systems need to be capable of dynamically personalizing, adapting and integrating web content and web services to create customized experiences for individual users. The goal of the research presented in this paper is to provide innovative techniques and technologies to achieve this. As part of this research, a novel architecture for the generation of Personalized Learning Activities, which highlights both the need for, and the power of combining and personalizing content and services has been developed. The system has been evaluated in the context of a Higher Education use case based on the user's perception of three key elements of the Personalized Learning Activity: the personalization, the usability and the educational benefits.
\end{abstract}

\section{Categories and Subject Descriptors}

H.5.4 [Hypertext/Hypermedia]: Architectures H.3.4 [Online Information Services]: Web-based services

\section{General Terms}

Algorithms, Design, Experimentation, Performance

\section{Keywords}

Adaptive Hypermedia, Personalization, Activity, Web as a Platform

\section{INTRODUCTION}

Permission to make digital or hard copies of all or part of this work for personal or classroom use is granted without fee provided that copies are not made or distributed for profit or commercial advantage and that copies bear this notice and the full citation on the first page. To copy otherwise, to republish, to post on servers or to redistribute to lists, requires prior specific permission and/or a fee.

i-KNOW'12, September 05-07, 2012, Graz, Austria.

Copyright 2012 ACM 978-1-4503-1242-4/12/09 ...\$15.00.
Web information system developers are increasingly seeking to apply 'Web as a Platform' based approaches where web based content is integrated with web services to provide the platform and environment features that were traditionally offered by desktop environments and applications [20]. Next generation personalized web information systems need to be capable of dynamically personalizing web content and web services to create customized experiences tailored to individual users.

The aim of personalizing web information systems is to tailor content (media) to the user's personal preferences, goals and context, which in turn increases the reusability of that content [6]. Personalization not only enhances the user's experience, but also assists web content and service providers in dynamically (and automatically) re-purposing their offerings for different users or contexts, facilitating reuse. Typically however, personalization as used in personalized search systems and information portals, tends to focus mainly on the selection and ranking of relevant content (web pages) or services [11].

Some adaptive web systems, particularly in application domains such as Education and Tourism have attempted to provide deeper, more sophisticated personalization [14][6]. However, they have predominantly concentrated on adaptive content selection, composition and navigation only [26]. Furthermore, the majority of such personalized systems tend to use bespoke or hand crafted content and services to support personalized applications. As such, the reuse of existing content and services still presents a significant problem.

The goal of the research presented in this paper is to provide innovative techniques and technologies to support the dynamic, integrated adaptivity and personalization of web media and services, enabling rich re-purposing of existing content and web services. As part of this research, a novel architecture for the generation of Personalized Learning Activities, which highlights both the need for, and the power of, combining and personalizing content and services, has been developed. This architecture is based on the 'Web as a Platform' approach where web-based content is integrated with web services to provide the platform and environment features that were traditionally offered by desktop environments and applications. As such, it represents a next generation personalized web information system, capable of both dynamically personalizing web media and web services to create customized experiences, such as Personalized Learning Activities, that are tailored to the needs, tasks and context of the user.

Such personalization and adaptivity must integrate the 
dynamic (automated) adaptation of web media resources together with the services that the user wishes to use. Thus next generation adaptivity will need to support:

- the seamless integration and dynamic adaptive presentation of web resources (content)

- the dynamic adaptive composition of web resources

- the dynamic adaptive composition of the web services

- the dynamic adaptation of web service behavior

This paper is structured as follows, a state of the art review of related work is first presented in Section 2. This review focuses on both the adaptive composition of media and the adaptive selection and composition of services. Additionally, the state of the art investigates current approaches to the design of Learning Activities. This is then followed, in Section 3, by a description of the AMASE Personalization Framework, which has been developed in order to support the adaptive composition of Personalized Learning Activities through the adaptive selection and sequencing of both multimedia content and services. Section 4 describes an evaluation of the AMASE Personalization Framework that was carried out and presents the results of that evaluation. The conclusions and future work are summarized in Section 5.

\subsection{Personalized Activities on the Web}

In order to provide authentic evaluation of this research, we have focussed on 'adaptive technology enhanced learning'. More specifically, the research is being applied in case studies based on authentic personalized learning scenarios in Higher Education. From an educational perspective, the design and implementation of 'Learning Activities' has been widely accepted as a means of providing greater learning engagement. Learning Activities consist of some form of $\operatorname{task}(\mathrm{s})$, associated services (tools) which could be used to perform the task(s), and appropriate learning content. Typically Learning Activities require some intuitive sequencing of operations, [9]. We refer to this sequencing as the workflow. Several popular (but limited) commercial environments have focused exclusively on supporting Learning Activities, e.g. LAMS [10]. Research into their pedagogic benefits have been investigated in several research projects e.g. LADiE [16] and DialogPlus [3].

Personalized Learning Activities provide all the opportunities of Learning Activities but with the significant advantages of content, services and workflow being dynamically adapted to benefit the learners. These benefits derive from the selection and customization of learning content, activities and learning sequences which more readily suit the particular contexts, needs and performance of the learner. This customization can be based on different 'dimensions' of the learning occurrence e.g. the learner's prior knowledge, competences, context, etc. [26]. Personalized Learning Activities typically comprise multiple (adaptive) learner tasks and (adaptive) learning content that can be linked together by an (adaptive) workflow. The size/duration (granularity) of a Personalized Learning Activity is defined by the activity designer (typically a tutor with some technical skills).

\section{RELATED WORK}

Traditional one-size-fits all hypermedia present all users with the same hypermedia document irrespective of their information needs. These issues can have the effect of increasing the cognitive load on a learner as they must not only attempt to learn the subject matter but also successfully navigate the hypermedia to find the most appropriate content. Adaptive Hypermedia (AH) [6] attempts to address these issues by adapting the hypermedia to the individual user, based on various properties of that user.

Systems such as GALE [22] and $\mathrm{ADAPT}^{2}$ [5] focus primarily on content and as such do not address the requirement for services. While $\mathrm{ADAPT}^{2}$ does support services, such as through its QuizGuide [23] component, this is still limited to features of the system rather than offering access to services openly available on the web. Additionally, these services are treated as a special type of content rather than as independent web services. This limits the type of control flow that can be used to sequence the services and hence limits the activity that can be implemented. For example, in $\mathrm{ADAPT}^{2}$ it is difficult to provide two services in parallel.

The IMS Learning Design (LD) specification [15] can be used to describe pedagogically driven Learning Activities using a platform independent language. The specification is broken into three levels; level A provides support for describing the basic structure of a static Learning Design that can be executed by a Learning Design player such as CopperCore [18] or learning management systems such as .LRN [12] and LAMS [10]. Learning Design provides basic support for adaptivity as part of the specification, and also supports the use of services as part of a Learning Activity.

However, the IMS LD Specification itself only supports three types of services: email, discussion and search, the implementation of which is platform dependent. Additional services can be added to LD by extending the specification with additional service types, but these would be specific to an individual platform as other LD platforms would not be able to handle the extensions. Various different approaches have been taken to address this issue such as that taken by LAMS. This drawback significantly limits LD as a specification for Learning Activities. Even if a specific platform does support additional services, the eLearning practitioner is still limited to a closed set of services rather than being free to use the services they find most appropriate for their activities. Furthermore, neither LD or systems such as LAMS support the adaptation of the services.

Where content adaptation research tends to focus entirely on content, adaptive service orchestration research has likewise focused exclusively on services. In workflow systems, adaptation and composition approaches are based on syntactic descriptions that combine other composite or elementary services with control constructs [24] to represent for example iterative, parallel and conditional execution. Such languages include Web Service Business Process Execution Language (WS-BPEL) [17] and Yet Another Workflow Language (YAWL) [25].

eFlow [7] is an example of a system that provides an approach for the dynamic composition, enactment and management of composite services. Adaptation is supported both at the level of the process definition (type) and the process instance (state). YAWL supports the dynamic selection of worklets [1] at runtime based on a set of rules that are written by the workflow designer. Context Aware Workflow Execution (CAWE) [2] is an adaptive workflow system that supports adaptation based on the individual user, the contextual properties and the device they are using. Finally, 
C-BPEL [13] supports the adaptive selection of services to instantiate the activities in a workflow at runtime.

There are also many approaches in which the adaptation and composition of services is based on semantic or ontological descriptions using languages such as Ontology Web Language for Services (OWL-S) [19] and Web Service Modeling Ontology (WSMO) [4]. The semantic/ontological descriptions enable the use of more flexible and dynamic techniques for the adaptation and composition of services. Another approach, often used in conjunction with semantic techniques, is the use of AI planning [21]. In this approach the composition problem corresponds to a state based planning problem, where a plan is devised to satisfy a goal by executing a sequence of services (actions) from a given initial state.

However, most of the workflow approaches outlined perform adaptations upon concrete implementations that are tied to specific technologies such as YAWL and WS-BPEL. This means that instructional designers need to be experts in order to design a complete and executable Learning Activity in these languages. Services and exchanged data are also hard bound to the workflow, therefore not allowing the dynamic resolution of tasks to services based on their abstracted descriptions. Consequently this limits their run time adaptation capabilities. There are also even less approaches allowing the dynamic adaptation of workflow instances based on the just in time evaluation of rules.

In addition most of these systems are built specifically to adapt or compose general workflows due to interoperability and availability problems of services. They do not consider adaptation from a personalization and customization perspective. Finally few approaches consider the domain specific core characteristics and design principles of the learning domain and learning activities.

\section{ARCHITECTURE}

To support the generation of Personalized Learning Activities that combine the personalized selection and presentation of multimedia content with adaptive selection and sequencing of user centric services in a unified manner a new system, AMASE, was developed. This system aims to combine state of the art techniques from both adaptive web and adaptive workflow systems and in so doing address the limitations of each respective approach with respect to the generation of personalized activities. This section describes the AMASE architecture, its components and how they interact with each other. Subsequently, the design and implementation of a web based portal for the delivery of Personalized Learning Activities is discussed.

\subsection{AMASE Personalisation Framework}

The AMASE architecture is based on a multi model approach in which the knowledge is pushed to the edges of the system and encapsulated in metadata models [8]. As shown in Figure 1, AMASE makes use of four models to support the personalization process.

- The Content Model contains information about the available multimedia content that the engine can use in the composition of a Personalized Learning Activity.

- The Service Model contains information about the available services that the engine can use in the composition of a Personalized Learning Activity.
- The Learner Model describes the goals, preferences, competencies, etc. of each learner.

- The Narrative Model encapsulates the strategy for composing the Personalized Learning Activity itself. It provides an abstract definition of an activity and adaptation rules that describe how the activity can be adapted to meet the needs of the individual learner. The Personalised Learning Activity is specified using Business Process Modeling Notation (BPMN) while the adaptation rules and models are written using the Drools rule language. The Narrative Model is defined is such a way as it remains abstract. It does not contain explicit references to any content resources or services but rather describes the activity in terms of the tasks of which it consists, the concepts that should be covered and the possible paths between the tasks.

Figure 1 provides a breakdown of the AMASE architecture and identifies the key components and their interactions when executing a Personalized Learning Activity. In order to generate an activity, the Narrative Model is executed by the Personalization Engine (PE), which reconciles the information available to the PE in the Learner Model with the the adaptation rules in order to generate an Abstract Personalized Activity. The Abstract Personalized Activity represents an instance of the Personalized Learning Activity defined in the Narrative Model. At this stage the control flow between the tasks that make up the activity has been personalized but the activity remains abstract as the appropriate content and services have not yet been selected in order to instantiate the tasks. In other words a personalized but abstract workflow is generated but specific content and services are not yet selected.

The next step is to deploy the Abstract Personalized Activity to the Enactment Engine so that it can be executed and made available to the Learner. The Enactment Engine is a jBPM based workflow engine, which executes the Personalized Learning Activities of each individual learner concurrently. The Enactment Engine also provides additional functionality such as logging and persistence of activities. This logging and persistence support enables the Personalized Learning Activity to be managed and saved/resumed by each user.

Once the Abstract Personalized Activity has been deployed to the Enactment Engine, it is then possible for a Learner to interact with their Personalized Learning Activity through the web based learning Portal. The Portal provides the Learner with an environment in which both the content and services that make up the activity are available in an integrated manner. As the Learner interacts with the Portal, requests are sent to the Enactment Engine in order to retrieve the appropriate content and services for the Learner. At this point the system makes requests to the Matchmaking/Composition service, which adaptively selects the appropriate content and services to meet the individual learners needs on a 'just in time' basis. The dynamic selections made by the Matchmaking/Composition service are again driven by the Narrative Model which describes how the adaptive selection should be carried out. In this way, the selection of appropriate content and services is aligned with the adaptation of the activity sequencing that has been carried out by the Personalization Engine but also suits the learner's current knowledge, skills, preferences, context. The 


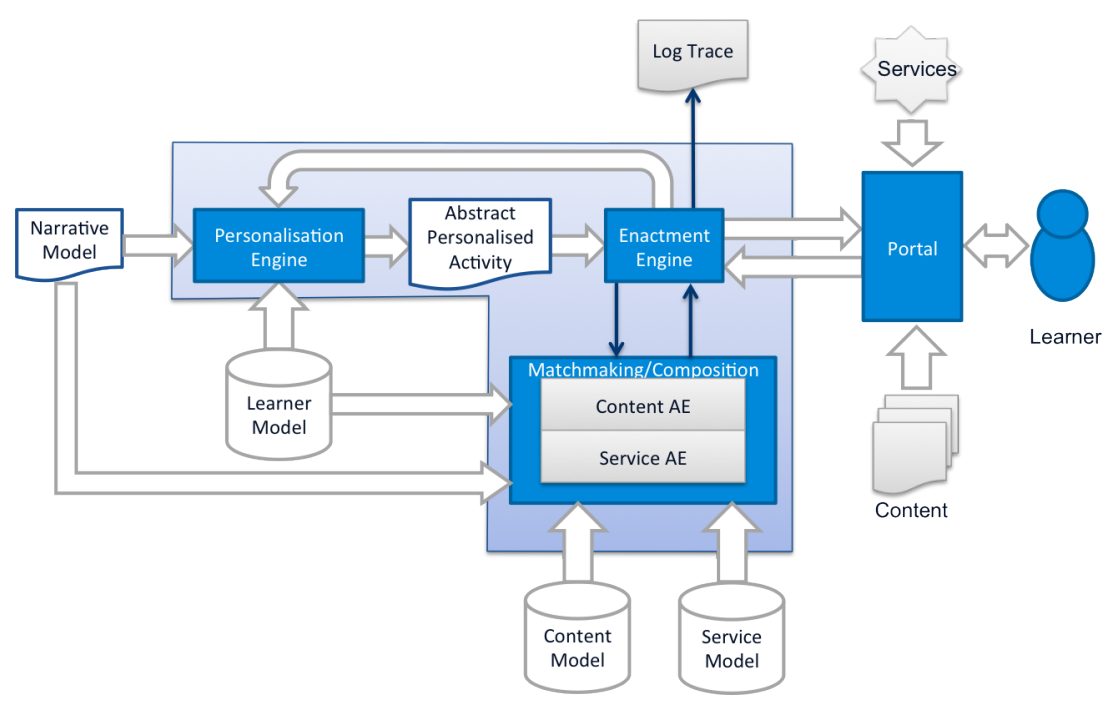

Figure 1: The AMASE Engine - Architectural Design and Components

Matchmaking/Composition service reconciles the information available from the Learner Model with information from the Content and Service Models, which describe the content and services that are available to the system.

\subsection{Personalized Learning Portal}

In order to make the functionality of this engine available to end users, it was first necessary to develop a portal through which learners could interact with the Personalized Learning Activities generated by AMASE. The portal is required to support the delivery of both multimedia content and interactive services, as well as aiding users as they progress through the activity. In this paper, we will focus on a particular deployment of AMASE which provides an online SQL Personalized Learning Activity as part of a 4th year undergraduate university course on relational databases, and the portal description and evaluation that follow relate to this.

The services used to implement the activities are developed using Java portlet APIs (JSR168 and JSR268). As such they can be deployed to any portal server that supports these APIs. For the Personalized Learning Activity delivery environment, the Liferay portal server was used. Liferay supports the publishing of portlets using unique URLs as a means of referencing specific instances, which can then be integrated into other environments.

Figures 2 and 3 provide screen captures of the Personalized SQL Course. The portal interface is divided into two main areas, the navigation menu on the left hand side of the screen and the content panel on the right hand side. The navigation menu consists of four sub menus:

1. The Overview menu provides a description of the personalized activity and information on how to use the system. It also includes a link to the requirements for the main project students had to undertake.

2. The My Course menu provides links to content that has been adaptively selected to meet the needs of the individual student.

3. The My Tasks menu provides access to the services

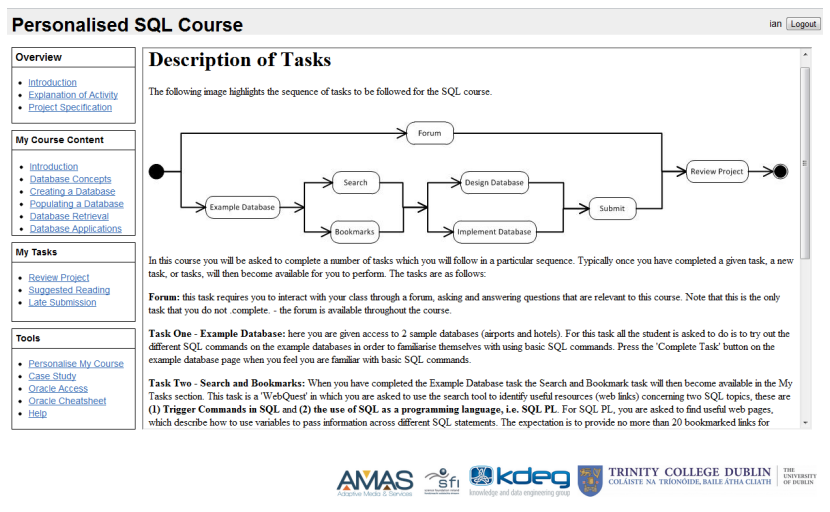

Figure 2: Screenshot from Personalized SQL Course showing home page

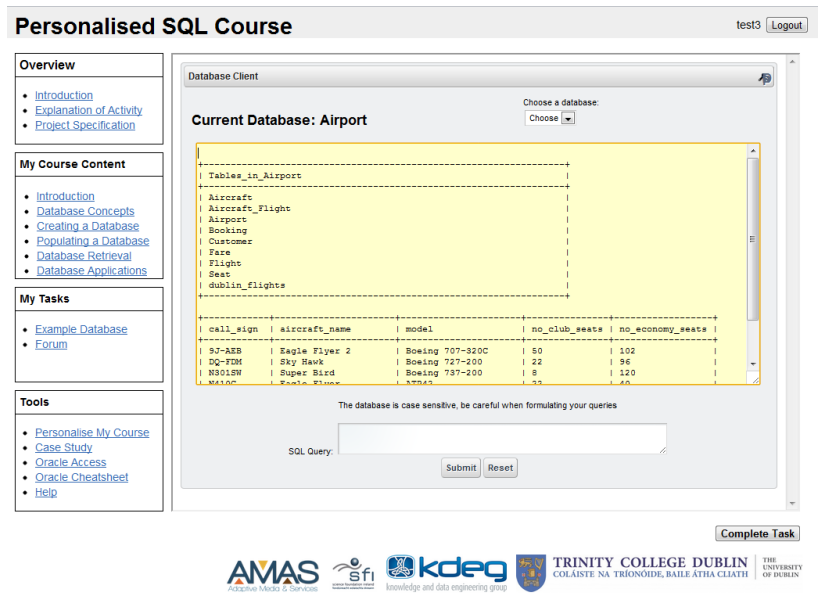

Figure 3: Screenshot from Personalized SQL Course showing service

from the activity workflow, which relate directly to the task that the student is currently performing. The 
activity is comprised of a number of tasks that the student must complete. Different services are made available here depending on the student's progress through the activity.

4. The Tools menu provides access to additional resources and information, including access to a tool that students can use to personalize their activity (by updating their responses to a short questionnaire about their SQL knowledge), as well as support material that the students might need to complete their project.

As shown in Figure 2 the content panel consists of a navigation menu for course subtopics, page navigation controls to allow students to move between pages of content in a section of the course and also the adaptively selected content itself. When a student selects a task to interact with, the content panel provides the student with access to the appropriate services as shown in Figure 3.

A task is implemented by one or more pieces of content and one or more services. A task can consist of the sequencing of services and content customized for the user. To realize the activity, a set of services was needed that could be used to instantiate the different tasks in the activity. In total 7 services were used (some were pre-existing Liferay services and some we developed ourselves).

- The Practice SQL Service is a sandbox environment in which students are free to try out different SQL commands that they learn during the course.

- The Forum Service provides an opportunity for interstudent and tutor-student discussion.

- The Search Service provides students with access to web searches that are integrated with the Bookmarking Service.

- The Bookmarking Service allows students to keep track of the links that they had found using the Search Service and to curate the bookmarked links prior to submitting them as part of the activity.

- The Submission Service allows students to upload their project reports to the system.

- The Review Service provides students with access to a set of reports that had been randomly allocated to them from the submitted documents.

- The Recommender Service encourages peer-to-peer learning amongst the students in the class.

\section{EVALUATION}

The evaluation of the personalized SQL activity had three key objectives: to investigate the user's perception of personalization; to investigate the clarity of the activity from a usability perspective; and to investigate the user's perception of the educational benefits of the personalized activity. The first step in carrying out this experiment was to design an appropriate Learning Activity that not only required the recomposition of multimedia content but also of different services. This activity also needed to incorporate adaptive behaviors in order to utilize the capabilities of the AMASE framework for the purposes of the evaluation. Figure 4 provides a graphical representation of the activity developed.
As shown, the activity consisted of 5 distinct tasks, and required different services for its realization.

- The first task was for the students to practice using SQL, (Practice SQL Service).

- The next task was a WebQuest task requiring the students to search the open web for content and to bookmark links that they considered appropriate, (Search and Bookmarking Services).

- The third task had the students designing and implementing an SQL database using Oracle, (completed outside the system using desktop-based tools).

- In the fourth task students had to write a report and submit it electronically, (Submission Service).

- The final task was a peer review in which the students were allocated a set of their peers' reports to review along with a review template, and then submit the reviews online, (Review Service)

Throughout the learning activity, students also had access to a Forum Service in which they could discuss any questions or problems they had during the activity with their peers. Finally, the students were also given a set of personalized recommendations of further reading compiled by the Recommender System Service. The recommendations were generated based on the relevant resources that each student bookmarked during the WebQuest task. More specifically, each student was recommended a set of suggested further reading which corresponded to resources that their peers had found relevant but that they themselves had not encountered.

In addition to the services provided for students to perform the required tasks, the students were also presented with personalized content covering the topic of SQL. Although shown as a single task in Figure 4 (Study SQL), this content was adaptively selected and sequenced for the students based on their prior knowledge of SQL.

\subsection{Experimental Setup}

The personalized SQL activity was used as part of a university course on relational databases taught to students from three different undergraduate degree courses, Computer Science, Computer Science and Linguistics and Computer Engineering. Students had access to the personalized SQL activity both from college computer labs and at home. The trial ran for a 16 week period and in total, 69 students used the online SQL activity.

To assess the students experience both during and after the learning activity an evaluation questionnaire was employed. The objective of the questionnaire was to gather opinions on the personalized generation of learning tasks within the SQL course. More specifically it elicited student opinion about the quality of the experience in both using the learning activities and the portal technologies. The evaluation questionnaire consisted of 26 questions in total covering various aspects of the system such as how often students used the system, the consistency of their experience, etc.

The next section discusses the evaluation results, focusing on the questionnaire results that have relevance to the evaluation of personalized activities in particular. Of the 69 


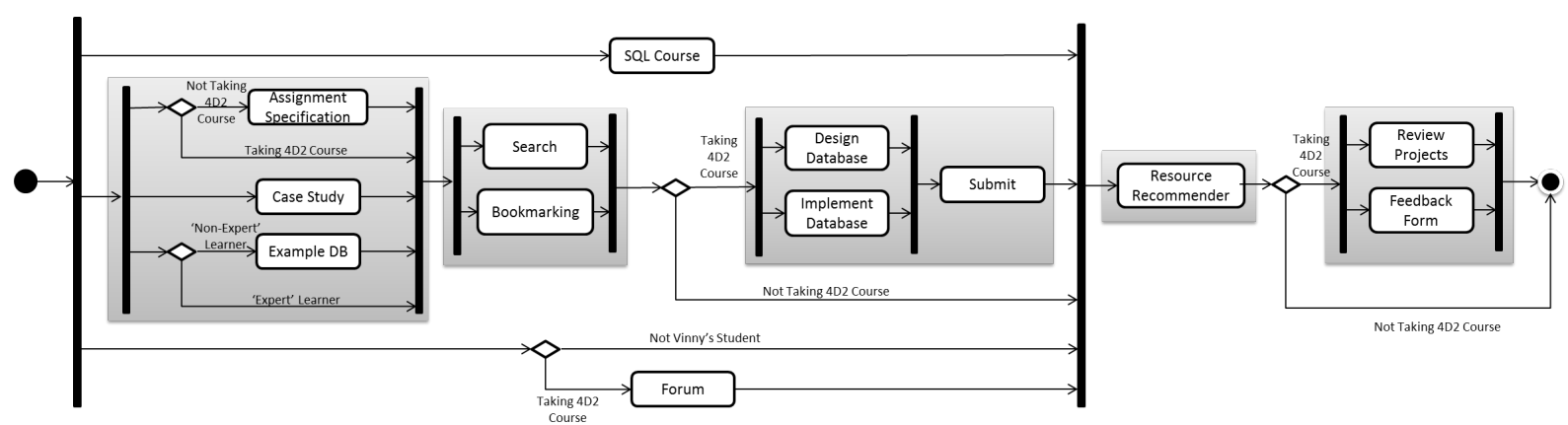

Figure 4: SQL Activity

students, only 32 were present on the day that the evaluation questionnaire was circulated. The questionnaire was given as a face to face paper survey at the beginning of one of the class lectures. The questions were categorized based on the objectives outlined earlier in this section. We now present results and discussion for each category.

\subsection{Results and Analysis}

\subsubsection{Students Perception of Personalization}

The questions in this category focused on how the students perceived the personalization carried out by the system. Students were asked to rate various aspects of the course affected by the adaptive selection and sequencing of content and services. Five questions fell into this category:

- Q1: Did the course(s) generated by the system reflect the answers you gave in the online course questionnaire?

- Q2: Were the courses generated easy to navigate?

- Q3: Did the course content of the generated course(s) appear disjoint?

- Q4: Did you find the flow between tasks appropriate?

- Q5: Did the course sections contain the content you expected?

The results for the five questions are shown in Figure 5 and Table 1. Questions 1 and 5 provide an indication of whether or not the adaptations carried out by the system were appropriate while questions 2, 3 and 4 look at the same aspect of the system from a slightly different perspective. By asking students about the ease of navigation and flow of the course we can identify whether or not the selection and sequencing carried out by the system are negatively impacting the course from the student's perspective. Any mistakes made by the system in the selection and sequencing would have a direct negative impact on these aspects of the course.

The results indicate that the students' perception of the personalization is very positive with an average positive response of $84.4 \%$. One aspect of the results that was less positive was whether or not the course content appeared disjoint (Q3). It is not immediately obvious why this is the case however it will be an aspect of the system that will be focused on during an additional, deeper analysis of the experimental results. The $10 \%$ who responded negatively when asked if the course sections contained the content they

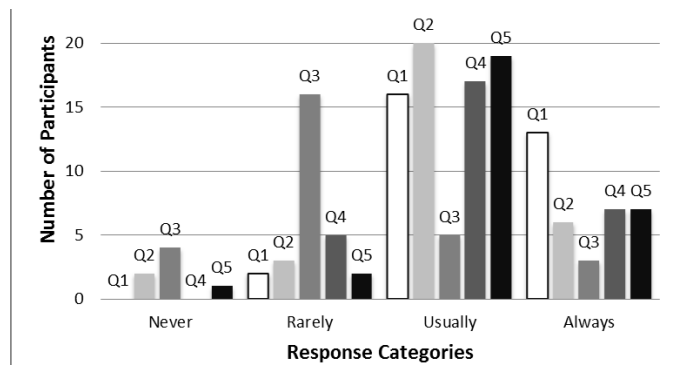

Figure 5: Students' Perception of Personalization

\begin{tabular}{|l|l|l|l|l|}
\hline Question & Never & Rarely & Usually & Always \\
\hline Q1 & 0 & 2 & 16 & 13 \\
\hline Q2 & 2 & 3 & 20 & 6 \\
\hline Q3 & 4 & 16 & 5 & 3 \\
\hline Q4 & 0 & 5 & 17 & 7 \\
\hline Q5 & 1 & 2 & 19 & 7 \\
\hline
\end{tabular}

Table 1: Students' Perception of Personalization

expected (Q5) can, in some part, be attributed to one specific section of the course on 'Database Applications', which is limited in the topics that are covered. Increasingly students are interested in this aspect of the course due to its role in web based applications and as such are beginning to expect more detail on this topic.

\subsubsection{Clarity of Usability}

This category focused on evaluating how clear the SQL activity was in conveying the objectives of the course and how intuitive the students found the process of interacting with the tasks. This category consisted of three questions:

- Q6: Were the objectives of the generated course(s) clear to you?

- Q7: Were the courses generated easy to navigate?

- Q8: Did you find interacting with the tasks intuitive?

In general the students responded positively to the questions in this category, as shown in Table 2 and Figure 6. However, a significant number of negative responses was recorded with respect to the clarity of the objectives (Q6). $35 \%$ of those who took part in the survey 'Rarely' felt that the objectives were clear to them. This is a significant proportion of the participants in the survey however it can, in 


\begin{tabular}{|l|l|l|l|l|}
\hline Question & Never & Rarely & Usually & Always \\
\hline Q6 & 0 & 8 & 20 & 4 \\
\hline Q7 & 2 & 3 & 20 & 6 \\
\hline Q8 & 0 & 8 & 18 & 2 \\
\hline
\end{tabular}

Table 2: Clarity of Usability

part, be attributed to some initial issues with the support material that was provided with the course. It was found, based on feedback from the students, that the introduction and overview material provided as part of the online activity was not sufficiently clear. This was subsequently rectified by providing significantly more detailed information. Similarly, not all students found the tasks intuitive (Q8); $29 \%$ stated that they 'Rarely' found the tasks intuitive. Again, based on some of the feedback from the class, this negative response can be in part attributed to limitations in the functionality of some of the services. For instance, there was an inconsistency between the Example Database data and the case study used in the course, which meant that when students tried to copy and paste commands from the text into to the service they did not work. By implementing some of the changes that were identified during the trialling of the SQL Course it should be possible to significantly improve the responses from students.

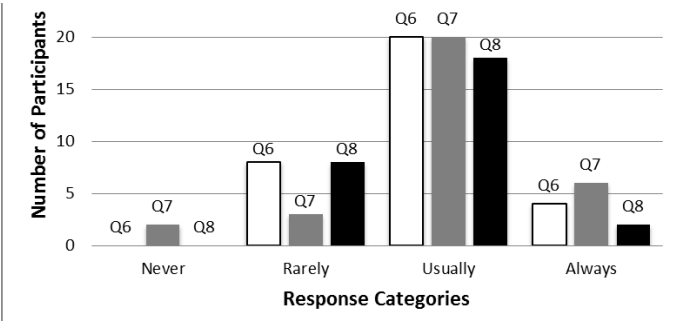

Figure 6: Clarity of Usability

\subsubsection{Perceived Assistance in Learning}

This category investigates the student's perceived value of the activity as a way of learning SQL and consisted of two questions:

- Q9: Do you agree that the activities helped you in learning the subjects covered by the course?

- Q10: Upon completion of the online course did you feel you had completed the objectives?

\begin{tabular}{|l|l|l|}
\hline Question & Yes & No \\
\hline Q9 & 24 & 6 \\
\hline
\end{tabular}

Table 3: Perceived Assistance in Learning (Q9)

\begin{tabular}{|l|l|l|l|l|}
\hline Question & $\begin{array}{l}\text { Strongly } \\
\text { Disagree }\end{array}$ & Disagree & Agree & $\begin{array}{l}\text { Strongly } \\
\text { Agree }\end{array}$ \\
\hline Q10 & 1 & 12 & 17 & 1 \\
\hline
\end{tabular}

Table 4: Perceived Assistance in Learning (Q10)
Evaluation results relating to the students' perception of personalization are summarized in Tables 3 and 4 and in Figure 7. As can be seen from the results, although the majority of students responded positively when asked if they found the activity helpful there was still a very significant $42 \%$ who did not find the activity helpful in their opinion (Q9). It is difficult to be definitive when interpreting this result without a more detailed evaluation, which is currently underway. However, several factors might be contributing to the high result. Some of the comments provided by students when answering the survey indicated that for some, the activity was simply seen as extra work that they had to do and that they would have preferred to simply be given a hand out containing all of the course content. In other cases, it was clear that initial technical difficulties that were experienced by students with the system had a significant impact on their perception of the course. A more positive result was found for the second question, which asked whether students felt that they had completed the objectives of the activity (Q10). $80 \%$ of students responded positively to this question, which would appear to support the argument made previously that students did not originally find the objectives of the course clear but that this issue was subsequently rectified. Despite this, it is still likely that the initial issues with the documentation provided with the course had an impact on the result here.

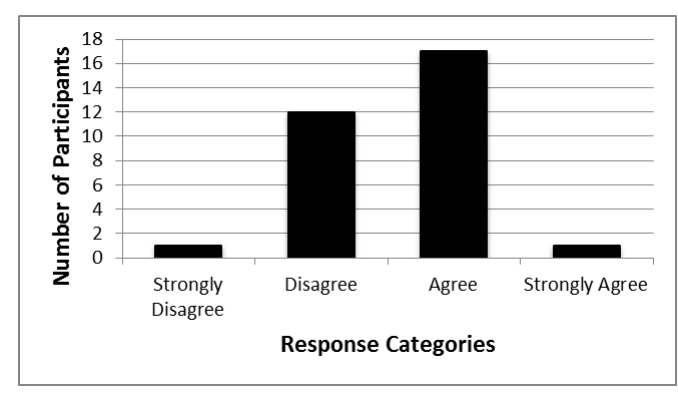

Figure 7: Perceived Assistance in Learning (Q10)

\section{CONCLUSION AND FUTURE WORK}

This paper describes the design and implementation of AMASE, a novel architecture capable of providing students with Personalized Learning Activities, which incorporates both dynamically personalized web media and web services. Such activities represent customized experiences tailored to the needs, tasks and context of the student. The paper also details the design and implementation of such a personalized activity, that was developed to help students learn SQL as part of a university course. The evaluation of the AMASE architecture through a user based trial in which the personalized SQL activity was provided to the students was then discussed and the results reported. The results show that the students found the personalization generally appropriate and that it did not affect the overall usability of the activity.

\section{ACKNOWLEDGEMENT}

This work was funded by Science Foundation Ireland via grant 08/IN.1/I2103 


\section{REFERENCES}

[1] Adams, M., ter Hofstede, A., Edmond, D. \& van der Aalst. Worklets: A service-oriented implementation of dynamic flexibility in workflows. In Proceedings of CoopIS 06 - On the Move to Meaningful Internet Systems: CoopIS, DOA, GADA, and ODBASE, pp.291-308, 2006.

[2] Ardissono, L., Furnari, R., Goy, A., Petrone, G. \& Segnan, M. The Context Aware Workflow Execution Framework. In International Journal of Autonomous and Adaptive Communications Systems, 5(1), Dargie, W., Kuwaharam N., \& Denko, M., (eds.), pp.58-76, 2012.

[3] Bailey, C., Zalfan, M. T., Davis, H. C., Fill, K. \& Conole, G. Panning for Gold: Designing Pedagogically-inspired Learning Nuggets. In Educational Technology \& Society, 9(1), pp.113-122, 2006.

[4] Bruijn, J.D., Bussler, C., Domingue, J. \& Fensel, D. Web Service Modeling Ontology (WSMO). W3C Member Submission, 2005. Available at: http://www.w3.org/Submission/WSMO/

[5] Brusilovsky, P., Sosnovsky, S. \& Yudelson, M. Ontology-based framework for user model interoperability in distributed learning environments. In Proceedings of World Conference on E-Learning in Corporate, Government, Healthcare, and Higher Education, (AACE E-Learn 2012), G. Richards, (ed.), pp. 2851-2855, 2005.

[6] Brusilovsky, P. Adaptive Navigation Support, In The Adaptive Web, P. Brusilovsky, A. Kobsa, W. Nejdl (eds.), LNCS vol. 4321, Springer, pp. 263-290, 2007.

[7] Casati, F., Ilnicki, S., Jin, L.J., Krishnamoorthy, V. \& Shan, M.C. Adaptive and dynamic service composition in eFlow. In Advanced Information Systems Engineering (CAiSE 2000). Wangler, B., \& Bergman, L.D. (eds.), LNCS vol. 1789, Springer, pp. 13-31, 2007.

[8] Conlan, O. \& Wade, V. Evaluation of APeLS - An Adaptive eLearning Service Based on the Multi-Model, Metadata-Driven Approach. In Proceedings of The Third International Conference on Adaptive Hypermedia and Adaptive Web-Based Systems (AH2004). P. De Bra \& W. Nejdl, (eds.), Springer, pp. 291-295, 2004.

[9] Dagger, D., Wade, V., \& Conlan, O. Personalisation for All: Making Adaptive Course Composition Easy. In Educational Technology \& Society, 8 (3), pp. 9-25, 2005.

[10] Dalziel, J. Implementing Learning Design: The Learning Activity Management System (LAMS). In Proceedings of The 20th Annual Conference of the Australasian Society for Computers in Learning in Tertiary Education (ASCILITE). G. Crisp et al., eds., Adelaide, Australia, pp. 593-596, 2003.

[11] Dou, X. Song, R. \& Wen, J. A Large-scale Evaluation and Analysis of Personalized Search Strategies, In Proceedings of 16th International Conference on World Wide Web (WWW16), Banff, Alberta, Canada, pp 581-590, 2007.

[12] Ecsobedo del Cid, J. P., de la Fuente Valentín, L., Gutiérrez, S., Pardo, A., Delgado Kloos, C. Implementation of a Learning Design Run-Time
Environment for the .LRN Learning Management System In Journal of Interactive Media in Education, special edition on Adaptation and IMS Learning Design, D. Burgos, (ed.) 2007(01), pp.1-12, 2007.

[13] Ghedira, C. \& Mezni, H. Through Personalized Web Service Composition Specification: From BPEL to C-BPEL. Electronic Notes in Theoretical Computer Science, 146(1), pp.117-132, 2006.

[14] Grapple. Generic Responsive Adaptive Personalized Learning Environment, EU FP7 Project, 2011. available at http://www.grapple-project.org/

[15] IMS. IMS Learning Design Specification, 2003. Available at: http://www.imsglobal.org.

[16] Jeffery, A., Conole, G. \& Falconer, I. LADiE Project Final Report, 2006. Available at: http://www.jisc.ac.uk/publications/reports/2006 /ladiefinalreport.aspx.

[17] Jordan, D. \& Evdemon, J. Web services business process execution language version 2.0. OASIS Standard, 2007.

[18] Martens, H. \& Vogten, H. A Reference Implementation of a Learning Design Engine. In Learning Design. R. Koper \& C. Tattersall, (eds.), Springer-Verlag Berlin/Heidelberg, pp. 91-108, 2005.

[19] Martin, D., Burstein, M., Hobbs, J., Lassila, O., McDermott, D., McIlraith, S., Narayanan, S., Paolucci, M., Parsia, B., Payne, T. \& others. OWL-S: Semantic markup for web services. W3C Member Submission, 2004.

[20] O' Reilly, T., 2005. What Is Web 2.0, http://oreilly.com/web2/archive/what-is-web-20.html, last accessed 17/02/12

[21] Rao, J., Su, X. A survey of automated web service composition methods. In: Proceedings of the First International Workshop on Semantic Web Services and Web Process Composition (SWSWPC), Springer, pp. 43-54, 2004.

[22] Smits, D. \& De Bra, P. GALE: a highly extensible adaptive hypermedia engine. In Proceedings of The 22nd ACM Conference on Hypertext and Hypermedia, Eindhoven, The Netherlands, pp. 63-72, 2011.

[23] Sosnovsky, S. Adaptive Navigation for Self-Assessment Quizzes. In Proceedings of the Third International Conference on Adaptive Hypermedia and Adaptive Web-Based Systems, W. Nejdl \& P. De Bra, (eds.), pp. 366-371, 2004.

[24] van Der Aalst, W.M.P., Ter Hofstede, A.H.M., Kiepuszewski, B. \& Barros, A.P. Workflow patterns. In Distributed and parallel databases, 14(1), Kluwer, pp.5-51, 2003.

[25] van Der Aalst, W.M.P. \& Ter Hofstede, A.H.M. YAWL: yet another workflow language. In Information Systems, 30(4), Elsevier, pp.245-275, 2005.

[26] Wade, V. Keynote Address: Challenges for the Multi-Dimensional Personalised Web, User Modelling, Adaptation and Personalisation (UMAP), Trento, Italy, 2009. 